\title{
lncRNA TSPEAR-AS2, a Novel Prognostic Biomarker, Promotes Oral Squamous Cell Carcinoma Progression by Upregulating PPM1A via Sponging miR-487a-3p
}

\author{
Yi-chao Xia, Jun Cao, Jing Yang, Ying Zhang, and Yong-sheng Li \\ Department of Oral and Maxillofacial Surgery, The First People's Hospital of Yunnan Province, The Affiliated Hospital of Kunming \\ University of Science and Technology, Kunming, Yunnan, China
}

Correspondence should be addressed to Yong-sheng Li; liyongshengkw@sohu.com

Received 10 June 2021; Accepted 4 July 2021; Published 19 July 2021

Academic Editor: Fu Wang

Copyright (c) 2021 Yi-chao Xia et al. This is an open access article distributed under the Creative Commons Attribution License, which permits unrestricted use, distribution, and reproduction in any medium, provided the original work is properly cited.

\begin{abstract}
Background. Long noncoding RNA (lncRNA) critically impacts the modulation of tumor developments and progressions. Our study is aimed at investigating the expressing patterns, clinical significance, and biological roles of lncRNA TSPEAR-AS2 (TSPEAR-AS2) in oral squamous cell carcinoma (OSCC). Material and Approach. The expressing states achieved by TSPEAR-AS2 were examined in OSCC specimens and cell lines by RT-PCR. The clinical significance of TSPEAR-AS2 was statistically analyzed. OSCC proliferating, invading, and migrating processes were examined with the use of wound healing assays, transwell, colony formation, and cell counting kit-8. Additionally, the downstream molecular mechanism of TSPEAR-AS2 in OSCC was explored. Results. TSPEAR-AS2 was overexpressed in OSCC tumors and cells. High TSPEAR-AS2 was associated with advanced TNM stage. Patients with high TSPEAR-AS2 expression displayed a shorter disease-free survival and total survival of OSCC patients than those with low TSPEAR-AS2 expressing level. It was found that knockdown of TSPEAR-AS2 could inhibit the proliferating, invading, and migrating processes pertaining to OSCC cells. Luciferase reporter tests and RNA pull-down results revealed that TSPEAR-AS2 enhanced the expressions of PPM1A by regulating miR-487a-3p, and TSPEAR-AS2 could be adopted as a miR-487a-3p sponge to inhibit PPM1A expression. Conclusion. Our study highlighted the significance of the TSPEAR-AS2/miR-487a-3p/PPM1A axis within OSCC progression and offered a novel biomarker and novel strategies for OSCC treatments.
\end{abstract}

\section{Introduction}

Oral squamous cell carcinoma (OSCC), ranking among the top eight causes of cancer-related death globally, takes up $>90 \%$ of head and neck cancer, affecting more than 400000 people every year $[1,2]$. The two main risk factors are alcohol and smoking for OSCC. Poor oral hygiene is also found to be involved in the aetiology of OSCC [3]. Despite the fact that great advances have been made in surgical techniques and chemoradiation therapy, the 5-year survival rate for OSCC patients remains very low $[4,5]$. The inability for diagnosing in the preliminary phase refers to the main reason for the unfavorable outcome of OSCC cases [6, 7]. It has been confirmed the involvements of multiple genetic and epigenetic abnormality in OSCC progressions, but the molecular mechanisms involved in OSCC tumorigenesis remain largely unclear.
Long noncoding RNAs (lncRNAs), with the transcription based on RNA polymerase II, are defined as transcripts containing $>200$ nucleotides [8]. More and more evidences have demonstrated that lncRNAs as one type of transcription factor display vital roles in diverse biological procedures $[9,10]$. The dysregulation of lncRNAs is also demonstrated with the involvement inside the occurrence and progression of several tumors $[11,12]$. For instance, overexpression of lncRNA PART1 promoted the proliferating and metastasis processes of lung carcinoma cells by sponging miR-17-5p [13]. IncRNA LINC00844, a highly expressed lncRNA in hepatocellular carcinoma, was shown to be a tumor inhibitor within hepatocellular carcinoma and suppress the metastasis of tumor cells via targeting AZGP1 [14]. In OSCC, IncRNA LINC01929 was demonstrated to strengthen the ability of OSCC cells in the proliferation and metastasis via targeting the miRNA- 
137-3p/FOXC1 axis [15]. Although a lot of human lncRNAs have been reported to be abnormally expressed in OSCC so far, the physiological functions of most lncRNAs have remained largely unclear.

Previously, lncRNA TSPEAR-AS2 was shown to display regulatory effects on hypoxia-induced pulmonary artery hypertension in cellular levels [16]. Recently, Ma and his group firstly reported TSPEAR-AS2 as a gastric cancerrelated lncRNA which served as an oncogene and suppressed the metastasis of tumor cells via suppressing GJA1 expression [17]. However, the potential functions of TSPEAR-AS2 in other tumors have not been investigated. In this study, we provided evidences that TSPEAR-AS2 was an overexpressed lncRNA in OSCC specimens and may be a novel biomarker for OSCC patients. In addition, we also studied the function of TSPEAR-AS2 in OSCC as well as its potential mechanisms.

\section{Materials and Methods}

2.1. Collection of Human Clinical Specimens. The researchers carried out the collection of ninety-five clinically-related OSCC tumor tissue and paired nearby nontumor tissue in the First People's Hospital of Yunnan Province. The tissue received the collection in the surgical process, and then it underwent the storage within liquid nitrogen or under the temperature of $-80^{\circ} \mathrm{C}$ to be employed subsequently. All the patients verified as OSCC according to histopathological evaluation were operated on at the First People's Hospital of Yunnan Province. This study was performed following a protocol approved by the Ethics Committee of The First People's Hospital of Yunnan Province. The respective patient provided written informed consent for participation.

2.2. Cell Culture and Transfection. The Cell Bank of the Chinese Academy of Sciences (Shanghai, China) offered six OSCC cell lines (SNU1041, FADU, HSU3, SCC25, SCC9, and SCC4) and the NHOK cell lines. All cells received the culture within DMEM (Invitrogen, Shenzhen, Guangdong, China) with 10\% FBS (Biocyto, Guangzhou, Guangdong, China) under the temperature of $37^{\circ} \mathrm{C}$ in $5 \% \mathrm{CO}_{2}$ and saturated humidity. Scrambled shRNA of TSPEAR-AS2 (sh-NC), TSPEAR-AS2 shRNAs (sh-TSPEAR-AS2-1 and sh-TSPEAR-AS2-2), miR-487a-3p mimic (NC mimics), and miR-487a-3p inhibitors (NC inhibitors) was purchased from GenePharma (China). Next, the mentioned received the transfection to SCC25 and FADU cell with the use of Lipofectamine 2000 (Invitrogen/Thermo Fisher Scientific).

2.3. RNA Extraction and RT-qPCR. The researchers adopted TRIzol reagent for obtaining overall RNA from specimens and cells. By the use of the PrimeScript RT reagent kit, $3 \mu \mathrm{g}$ RNA overall received the reverse transcription to cDNA. RT-qPCR was performed with the use of FastStart Universal SYBR Green Master (Roche, Pudong, Shanghai, China) on a Bio-Rad RT-PCR cycler (Roche, Pudong, Shanghai, China). GAPDH and U6 became the control to normalize mRNA's and miRNA's expressing levels, separately. Relative expres- sion values of genes were analyzed by the $2^{-\Delta \Delta \mathrm{Ct}}$ approach. Table 1 lists the primer sequence of RNAs.

2.4. Cell Counting Kit-8 (CCK-8) Assay. To analyze the growth of the OSCC cell lines, the CCK- 8 test (Dojindo Laboratories) was performed by complying with the producer's protocol. OSCC cells were cultured in 96-well plates overnight. After $1 \mathrm{~d}, 2 \mathrm{~d}, 3 \mathrm{~d}$, and $4 \mathrm{~d}$, cells were added with CCK-8 (Lifusai, Nanjing, Jiangsu, China). Optical density at $490 \mathrm{~nm}$ was measured using a microplate reader, and data were expressed as absorbance values. GraphPad Prism 8 software (GraphPad Software, Inc.) was used to plot the cell growth curve. The tests were carried out no less than 3 times.

2.5. Colony Formation Assays. $0.5 \times 10^{3}$ cells received the inoculation to a 12 -well plate and then the 10 -day culture. Fresh medium on the 5th day was used to replace the original medium. After the incubating process, the researchers adopted PBS for rinsing cells. Next, the $5 \mathrm{~min}$ immobilizing process for cells was carried out by using 4\% paraformaldehyde, and then the $30 \mathrm{~s}$ staining process by using $0.1 \%$ crystal violet was used. Finally, the counting and recording processes were used for the number of colonies (over 50 cells).

2.6. EdU Incorporation Assays. Cells received the culture within 24-well plates, and the researchers introduced $10 \mu \mathrm{M}$ EdU to each well. Subsequently, 4\% formaldehyde was used to fix the cells for $30 \mathrm{~min}$. $48 \mathrm{~h}$ later, $50 \mu \mathrm{M}$ of EdU labeling medium was introduced to the respective well, and the cell received the $8 \mathrm{~h}$ incubation. Next, the cells were stained with Hoechst 33342 for $20 \mathrm{~min}$ and were captured. Eventually, under the microscope, EdU-positive cells received the observation and the counting process.

2.7. Wound Healing Assay. The researchers carried out the seeding and culturing process for the cell within a confluent monolayer in a rectangular cell culture plate. The cell received the culture till the confluence reaching nearly $100 \%$. $10 \mathrm{~L}$ pipette tips were used to create cell wounds. Moreover, in FBS-free F12K medium (Procell, Nanjing, Jiangsu, China), cells were then cultivated. At $24 \mathrm{~h}$ after culture, an inverted microscope was used to measure the width of the wounds. Gap distance was quantified using NIH ImageJ software version 1.50.

2.8. Transwell Assay. Using transwell chambers, the ability of cell invasion was examined. A total of $2 \times 10^{4}$ cells was supplemented to the upper compartment of each transwell chamber (pore size: $8 \mu \mathrm{M}$; Corning, Haidian, Beijing, China), and $600 \mu \mathrm{L}$ of medium containing $10 \%$ FBS received the addition into the compartment which was relatively low. When $24 \mathrm{~h}$ incubating process was achieved under room temperature, the researchers employed a cotton swab for scraping cells on the upper chamber's internal surface. In the chamber which was relatively low, ethyl alcohol was applied for fixing the invaded cell. Next, $0.1 \%$ crystal violet was applied to stain the collect cells for $15 \mathrm{~min}$. Cell number was manually counted. 
TABLE 1: The primers used in this study for RT-PCR.

\begin{tabular}{lc}
\hline Names & Sequences $\left(5^{\prime}-3^{\prime}\right)$ \\
\hline TSPEAR-AS2: F & ACCCTCGACGTCCGTCCACGG \\
TSPEAR-AS2: R & GCAGGCCATGCAAGTCACAG \\
miR-487a-3p: F & ATGGCGGAATCATACAGGGAC \\
miR-487a-3p: R & CTCAACTGGTGTCGTGGAGTC \\
PPM1A: F & AGGGGCAGGGTAATGGGTT \\
PPM1A: R & GATCACAGCCGTATGTGCATC \\
GAPDH: F & GGAGCGAGATCCCTCCAAAAT \\
GAPDH: R & GGCTGTTGTCATACTTCTCATGG \\
U6: F & GCGCGTCGTGAAGCGTTC \\
U6: R & GTGCAGGGTCCGAGGT \\
\hline
\end{tabular}

2.9. Subcellular Fractionation. Cytoplasmic and nuclear separations were carried out with the use of PARIS Kit (Life Technologies, Hangzhou, Zhejiang, China) based on the producer's guideline.

2.10. RNA Pull-Down Assay. Biotinylated TSPEAR-AS2 probe, $\mathrm{miR}-487 \mathrm{a}-3 \mathrm{p}$ probe, and the relative control were obtained according to GenePharma (Shanghai, China). Cellular lysates were combined with M-280 streptavidin magnetic beads (Invitrogen) as discussed in prior studies [18], and qRT-PCR was used to detect the TSPEAR-AS2 or miR-487a-3 expression.

2.11. Luciferase Reporter Assay. TSPEAR-AS2-WT/MUT or PPM1A-WT/MUT was subcloned into the pmirGLO dualluciferase vector (Biomart, Haidian, Beijing, China). First, SCC25 or FADU cells received the cotransfection by using pmirGLO-TSPEAR-AS2-WT/MUT and NC mimics or miR-487a-3p mimics. Second, SCC25 or FADU cells received the cotransfection by using miR-487a-3p mimics and pmirGLO-PPM1A-WT/MUT. Luciferase and renilla signals received the measurement $48 \mathrm{~h}$ after being transfected with the use of the Dual-Luciferase Reporter Assay System (Promega, Pudong, Shanghai, China). Luciferase reporter assays were conducted in triplicate.

2.12. Western Blot Assay. SCC25 and FADU cells were harvested and lysed in RIPA Lysis Buffer (Yita Biology, Pinggu, Beijing, China) to collect proteins. Proteins were separated using SDS-PAGE at $30 \mu \mathrm{g} / \mathrm{lane}$ and transferred to a nitrocellulose membrane (EMD Millipore, Pudong, Shanghai, China). Next, our group blocked the membrane with $5 \%$ dried skimmed milk for $1 \mathrm{~h}$. Then, the membrane was incubated with primary antibodies including anti-PPM1A (Cat no. ab14824, Abcam) and anti-GAPHD (Cat no. ab8245; Abcam) at $4^{\circ} \mathrm{C}$ throughout the night and with horseradish peroxidase- (HRP-) conjugated secondary antibodies (Guduo, Shanghai, China) for $1 \mathrm{~h}$ under ambient temperatures. The researchers employed ImageJ software for quantifying the density exhibited by the respective band.

2.13. Statistical Analysis. Data had the presentation of mean \pm standard deviation (SD). A statistic-based investigation was conducted with the use of SPSS (IBM, Armonk, NY,
USA) and diagrams received the mapping process with the use of GraphPad Prism software. For comparison within different groups, Student's $t$-test or one-way ANOVA was performed. The OS and DFS were analyzed by log-rank test, and survival curves were plotted according to Kaplan-Meier. The Cox proportional hazards model was employed for the multivariate analysis. The statistical significance $(P$ value) is set as less than 0.05 .

\section{Results}

3.1. Expression of TSPEAR-AS2 in OSCC Tissues and Adjacent Normal Tissues. For exploring the potential role of TSPEAR-AS2 in OSCC, the expression of TSPEAR-AS2 was analyzed in OSCC tissues and cell lines. As shown in Figure 1(a), compared with normal group, the expression of TSPEAR-AS2 was distinctly increased in OSCC tissues $(P<0.05)$. ROC assays showed strong separation between the two groups (tumor group vs. normal group), with an AUC of 0.8114 (0.7478-0.8749) (Figure 1(b)). In addition, higher levels of TSPEAR-AS2 were observed in OSCC specimens with III-IV compared with those with I-II (Figure 1(c)). ROC assays showed strong separation between the two groups (I-II vs. III-IV), with an AUC of 0.8387 (0.75500.9225 ) (Figure 1(d)). Furthermore, our group also observed that TSPEAR-AS2 expression was distinctly upregulated in six OSCC cells compared with NHOK cells (Figure 1(e)).

3.2. The Prognostic Value of TSPEAR-AS2 Expression in OSCC Patients. To better understand the potential roles of TSPEAR-AS2 in OSCC development, the patients were divided into high and low expression groups by the median expression level of TSPEAR-AS2 (5.89). The Chi-square test revealed that high TSPEAR-AS2 expression was associated with the advanced TNM stage $(P=0.022)$ (Table 2$)$. However, there was no association between TSPEAR-AS2 expression and other clinical factors (all $P>0.05$ ). With five-year follow-up by the apartment of our hospital, we collected five-year survival data, which was analyzed by KaplanMeier analysis and log-rank test. We found that the patients in the high TSPEAR-AS2 expression group had shorter overall survival (OS, $P=0.0120$, Figure $1(\mathrm{f})$ ) and disease-free survival (DFS, $P=0.0008$, Figure $1(\mathrm{~g})$ ) than those in the low TSPEAR-AS2 expression group. More importantly, after multivariate analyses of prognostic factors in OSCC patients, the TSPEAR-AS2 expression level was identified to be an independent prognostic factor for OS $(\mathrm{HR}=2.893,95 \% \mathrm{CI}$ : $1.217-4.324 ; P=0.014)$, as well as DFS ( $\mathrm{HR}=3.015,95 \%$ CI: $1.334-4.732 ; P=0.007$ ) of OSCC patients (Table 3 ).

3.3. The Effects of TSPEAR-AS2 Knockdown in OSCC Progression. For exploring the regulating effect exerted by TSPEAR-AS2 in OSCC cells, three shRNAs (sh-TSPEARAS2-1 and sh-TSPEAR-AS2-2) targeted to TSPEAR-AS2 and one scrambled control shRNA (sh-NC) were applied. The efficiency was then determined in stably transfected cells by RT-qPCR (Figure 2(a)). CCK-8 results showed that the proliferation of TSPEAR-AS2 knockdown cells received the significant inhibition in contrast to the negative control and 


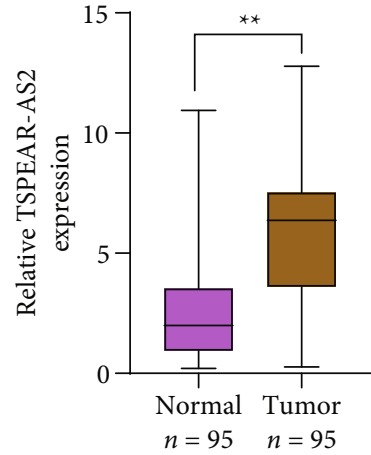

(a)

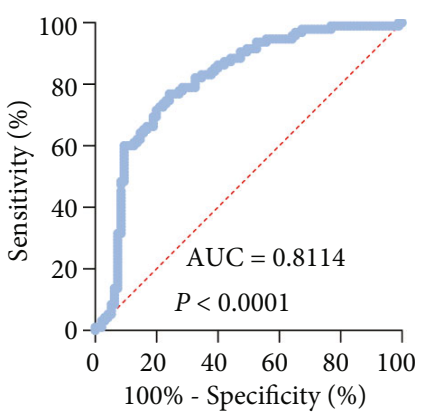

(b)

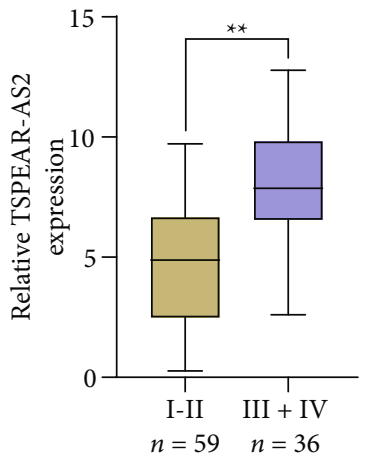

(c)

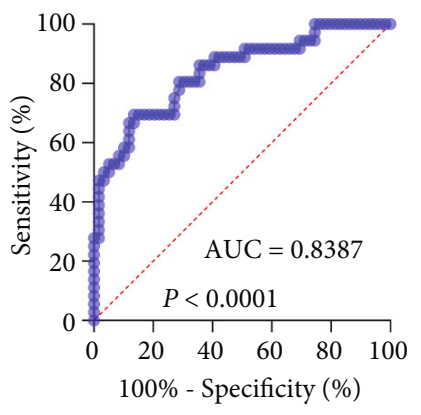

(d)

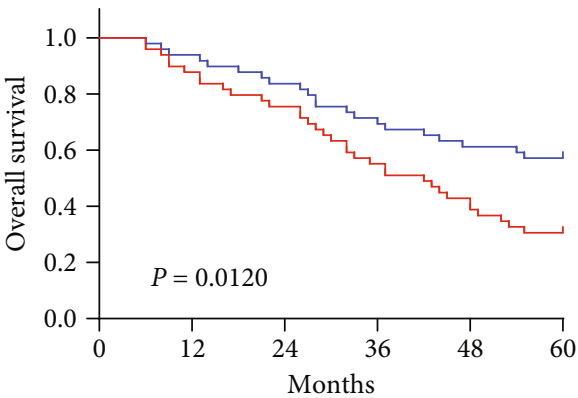

- Low TSPEAR-AS2 expression

$\rightarrow$ High TSPEAR-AS2 expression

(f)

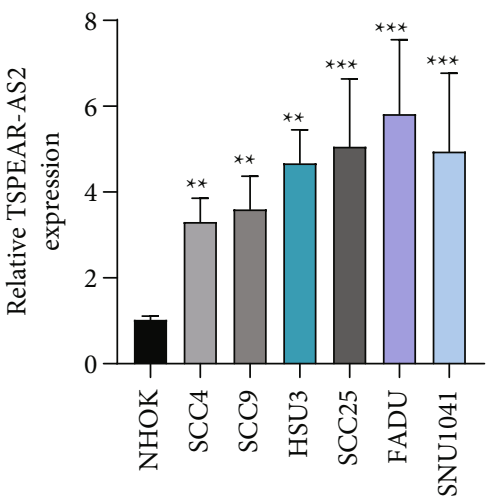

(e)

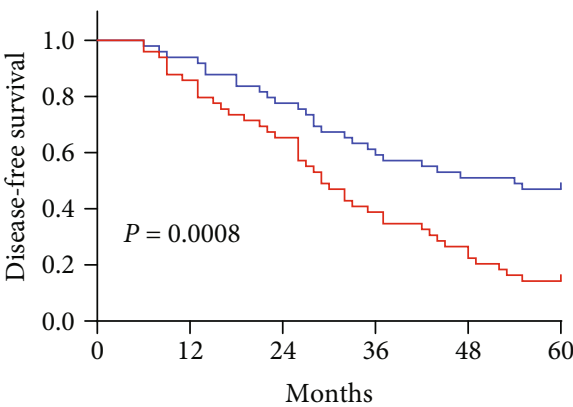

- Low TSPEAR-AS2 expression

- High TSPEAR-AS2 expression

(g)

FIgURE 1: The distinct upregulation of TSPEAR-AS2 in OSCC patients and its clinical significance. (a) TSPEAR-AS2 level was distinctly greater within OSCC tissue as compared with that in normal tissues. (b) ROC curve analysis for the detection of CRC using TSPEAR-AS2. (c) The comparison of TSPEAR-AS2 levels in OSCC specimens with I-II or III-IV. (d) TSPEAR-AS2 can be used to distinguishing OSCC specimens with III-IV from those with I-II. (e) Relative TSPEAR-AS2 expression was measured by qRT-PCR in six OSCC cells and NHOK cells. ( $\mathrm{f}$ and g) The correlation of TSPEAR-AS2 expression with OS (f) and DFS (g) of OSCC patients analyzed by Kaplan-Meier analysis. ${ }^{* * *} P<0.001,{ }^{* *} P<0.01$.

the blank group in both SCC25 and FADU cell lines (Figure 2(b)). Colony formation assay and Edu assays also showed that knockdown of TSPEAR-AS2 considerably suppressed the viability of SCC25 and FADU cells (Figures 2(c) and 2(d)). Subsequently, we performed wound healing assay and transwell assay to explore the effects of TSPEAR-AS2 knockdown on metastatic abilities of OSCC cells. It was observed that knockdown of TSPEAR-AS2 visibly reduced the migrative (Figure 3(a)) and invasive (Figures 3(b) and $3(\mathrm{c}))$ abilities of SCC25 and FADU.

3.4. TSPEAR-AS2 Served as a Sponge of miR-487a-3p. It has been demonstrated that numerous cytoplasmic lncRNAs have been reported to be competing endogenous RNAs (ceRNAs) through the competitive bind process of microRNAs $[19,20]$. With the use of the subcellular fractionating 
TABLE 2: Relationship between lncRNA TSPEAR-AS2 expression and clinicopathological characteristics.

\begin{tabular}{lcccc}
\hline & \multicolumn{4}{c}{ TSPEAR- } \\
Variable & Cases $(n)$ & $\begin{array}{c}\text { AS2 } \\
\text { expression } \\
\text { High }\end{array}$ & Low & \\
& & & & 0.745 \\
Age & 45 & 24 & 21 & \\
$\quad<60$ & 50 & 25 & 25 & \\
$\quad>60$ & & & & 0.972 \\
Gender & 58 & 30 & 28 & \\
$\quad$ Male & 37 & 19 & 18 & \\
$\quad$ Female & & & & 0.082 \\
Histology/differentiation & 57 & 26 & 31 & \\
$\quad$ Well + moderate & 38 & 23 & 15 & \\
$\quad$ Poor & & & & 0.022 \\
TNM stage & 59 & 25 & 34 & \\
$\quad$ I + II & 36 & 24 & 12 & \\
$\quad$ III + IV & & & & \\
\hline
\end{tabular}

process, TSPEAR-AS2 had the expression within the nucleus and cytoplasm, and a greater proportion of TSPEAR-AS2 was observed in the cytoplasm (Figure 4(a)). Bioinformatics tools estimated the complementary binding site in TSPEAR-AS2 and miR-487a-3p, which was confirmed using the luciferase reporter assay (Figures 4(b) and 4(c)). RNA pull-down assays also demonstrated the combination between TSPEAR-AS2 and miR-487a-3p (Figure 4(d)). Finally, RT-PCR assays revealed that after TSPEAR-AS2 expression was suppressed, the levels of miR-487a-3p were increased in SCC25 and FADU (Figure 4(e)).

3.5. PPM1A Was Identified as a Direct Target of miR-487a-3p in OSCC Cells. To explore the specific mechanism of the TSPEAR-AS2/miR-487a-3p axis, we predicted the potential targets of miR-487a-3p by using TargetScan. The results showed that PPM1A might be a potential target of miR487a-3p (Figure 5(a)), which was demonstrated with the use of luciferase reporter assays (Figure 5(b)). In addition, we observed that miR-487a-3p overexpression distinctly suppressed the levels of TSPEAR-AS2 and PPM1A, while miR-487a-3p knockdown displayed an opposite effect (Figure 5(c)). Further rescue experiments revealed that knockdown of miR-487a-3p distinctly reversed the suppression of TSPEAR-AS2 knockdown on the expression of PPM1A (Figure 5(d)) in FADU cells.

\section{Discussion}

The identification of sensitive biomarkers was very important for the improvements of the clinical outcome of OSCC patients $[21,22]$. There have been many papers reporting the discovery of OSCC biomarkers, but only a few biomarkers have been validated and successfully applied in routine clinical practice $[23,24]$. Moreover, most biomarkers possess limitations for the early detection of OSCC, and their prognostic value was plagued by inaccuracies. In recent years, more and more studies highlighted the potential of lncRNAs used as novel biomarkers for cancer patients [25, 26]. Several lncRNAs, such as IncRNA HOXA11-AS, IncRNA CASC9, and lncRNA LEF1-AS1, have been shown to possess diagnostic and prognostic values for OSCC patients [27-29]. In this study, we identified a novel OSCC-related lncRNA, TSPEAR-AS2 which was highly expressed in OSCC and could be used as a diagnostic and prognostic marker for OSCC patients. The OS and DFS of OSCC patients with high TSPEAR-AS2 expression were distinctly shorter than those with low TSPEAR-AS2 expression, which was consistent with the prognostic value of TSPEAR-AS2 expression in gastric cancer patients [17]. However, the sample size was relatively small. We will collect more samples for research in the future.

Because of the role of cell signaling pathways in cancer initiation, progression, and metastasis, lncRNAs involved in these pathways can influence all aspects of tumorigenesis $[30,31]$. Therefore, IncRNAs may play a role in carcinogenesis or tumor inhibition. For instance, suppression of TTNAS1 resulted in an ability inhibition of OSCC cells in the tumor growth and metastasis via miR-411-3p/NFAT5 axis [32]. Overexpression of MCM3AP-AS1 promoted the proliferation and invasion of OSCC cells via regulating miR-204-5p/FOXC1 [33]. Given that TSPEAR-AS2 was highly expressed in OSCC specimens and predicted a poor prognosis of OSCC patients, we performed loss-of-function assays, finding that knockdown of TSPEAR-AS2 distinctly suppressed the proliferation, migration, and invasion of OSCC cells. In the future, in vivo assays were needed to further demonstrate the effects of TSPEAR-AS2 on OSCC progression. Previously, the similar oncogenic roles of TSPEAR-AS2 on gastric cancer cells were also demonstrated, suggesting the great potential of TSPEAR-AS2 used as a novel therapeutic target [17].

As revealed in existing research, miRNA is carcinogenic or inhibitory within tumorigenesis, and the expressions achieved by $\operatorname{lncRNAs}$ are able to control the activities of miRNAs [34, 35]. Increasing evidences show that lncRNAs control OSCC to develop and progress by sponging an array of downstream miRNAs [36, 37]. Thus, delving into the mentioned miRNAs can help develop feasible approaches for preventing and treating the OSCC. Our experiments demonstrated TSPEAR-AS2 had a major expression within the cytoplasm, suggesting the tremendous possibility of TSPEAR-AS2 acting as a ceRNA. Starbase 2.0 revealed miR-487a-3p may be a target of TSPEAR-AS2, which was further confirmed by Luciferase Reporter Gene Assay, RNA pull-down, and RT-PCR. Previously, miR-487a-3p has been reported to be overexpressed in several tumors, such as colon cancer and gastric cancer [38, 39]. Importantly, in OSCC, miR-487a-3p was found to display an upregulated level and suppress the proliferation and metastasis of OSCC cells [40]. These findings suggested TSPEAR-AS2 may display its oncogenic roles via sponging miR-487a-3p.

PPM1A refers to a protein phosphatase $2 \mathrm{C}$ family member of Ser/Thr protein phosphatases [41]. It can control TGF-beta/Smad19-21 and mitogen-activated protein kinase22 cellular signaling channels, and proliferating, invading, and 
TABLE 3: Multivariate analyses of prognostic factors in OSCC patients.

\begin{tabular}{lccccc}
\hline Variables & HR & Overall survival & & \multicolumn{2}{c}{ Disease-free survival } \\
& 95\% CI & $P$ value & HR & 0.821 & $0.445-1.532$ \\
\hline Age & 0.783 & $0.453-1.343$ & 0.459 & 0.671 & $0.445-1.435$ \\
Gender & 0.556 & $0.341-1.231$ & 0.244 & 0.321 \\
Histology/differentiation & 1.132 & $0.673-1.873$ & 0.112 & 1.345 & $0.792-1.832$ \\
TNM stage & 3.132 & $1.325-4.789$ & 0.005 & 3.436 & $1.429-5.554$ \\
TSPEAR-AS2 expression & 2.893 & $1.217-4.324$ & 0.014 & 3.015 & $1.334-4.732$ \\
\hline
\end{tabular}

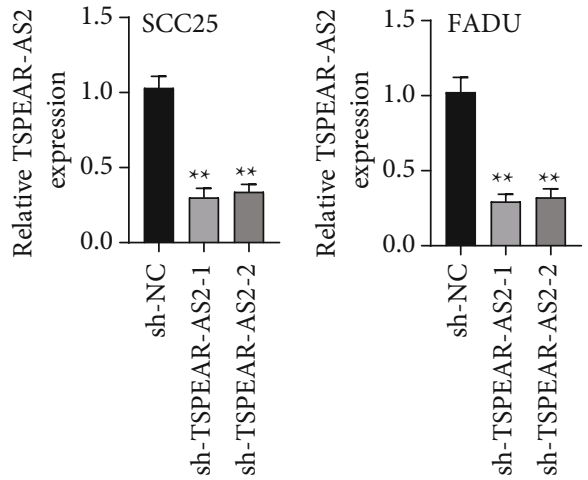

(a)

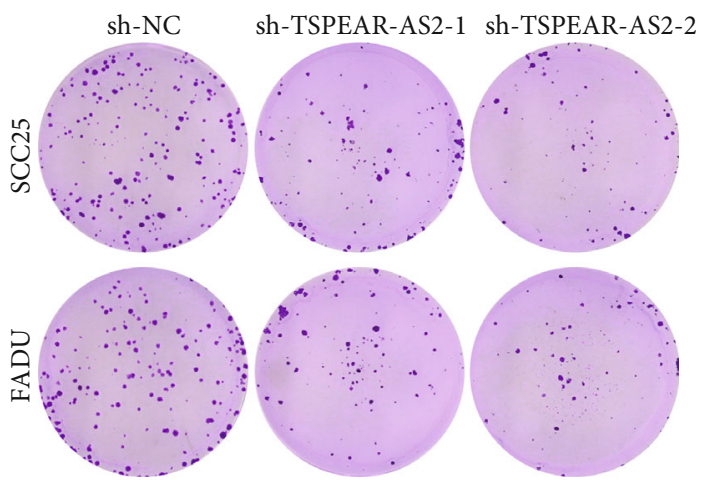

(c)

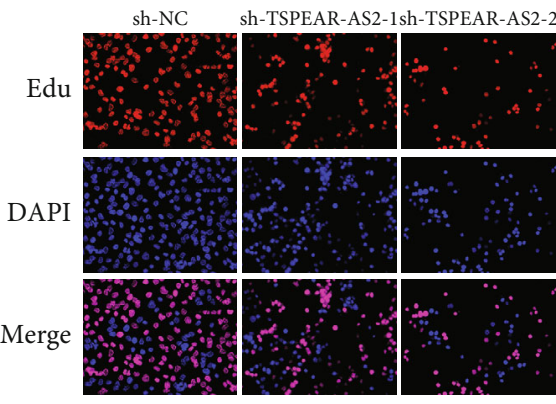

SCC25

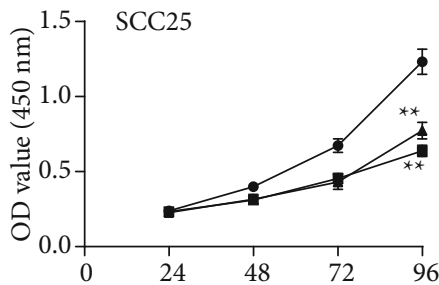

(h)

- sh-NC

- sh-TSPEAR-AS2-1

- sh-TSPEAR-AS2-2

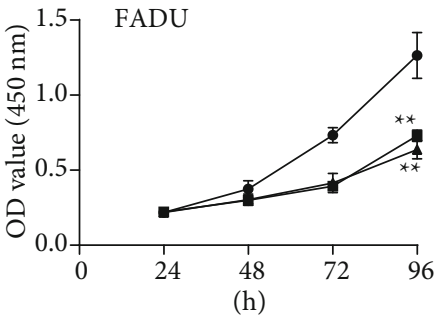

- sh-NC

- sh-TSPEAR-AS2-1

- sh-TSPEAR-AS2-2

(b)

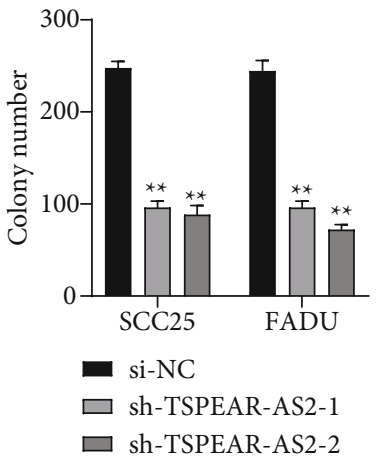

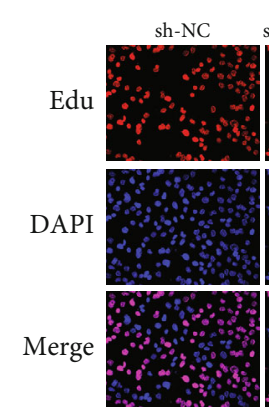

(d)

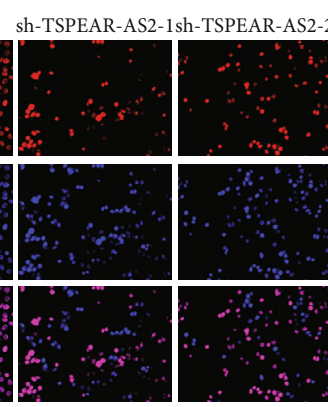

FADU

FIgURE 2: TSPEAR-AS2 depletion inhibits OSCC proliferation. (a) Relative expression of TSPEAR-AS2 after transfection with shRNAs. (b) CCK8 assay for cell proliferation analysis. (c) Colony formation test was carried out for cell proliferation after transfection of sh-TSPEARAS2-1 or sh-TSPEAR-AS2-2. (d) TSPEAR-AS2 knockdown inhibited cell proliferation, as determined by EdU assays. ${ }^{* *} P<0.01$.

migrating processes of cells $[42,43]$. In OSCC, PPM1A has been reported to be highly expressed and promoted the proliferation and metastasis of OSCC cells, whereas how PPM1A controls the mentioned activities requires in-depth studies [40]. In this study, we found PPM1A may be a target of miR-487a-3p. Overexpressed miR-487a-3p suppressed the 


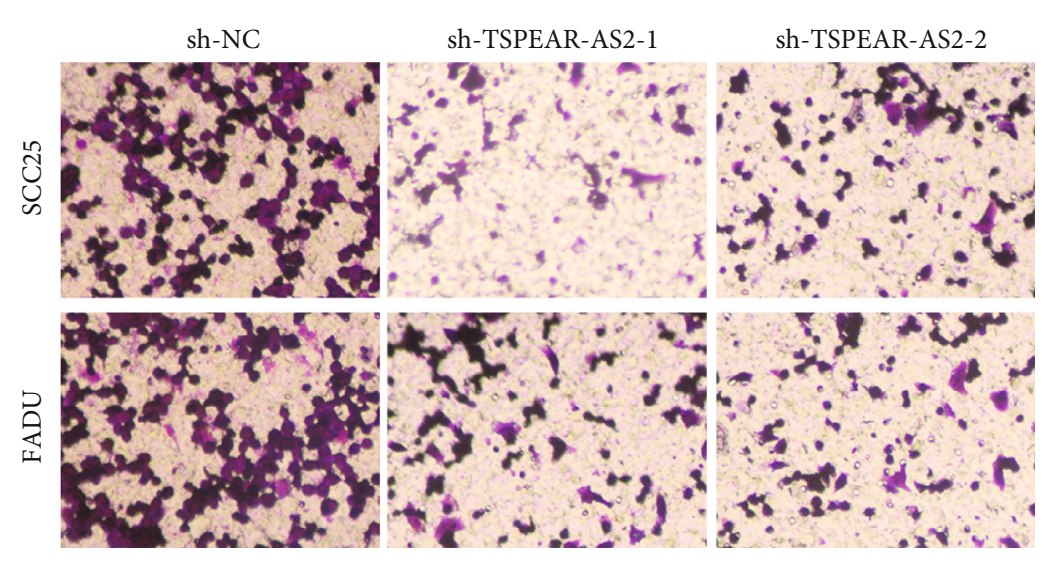

(a)

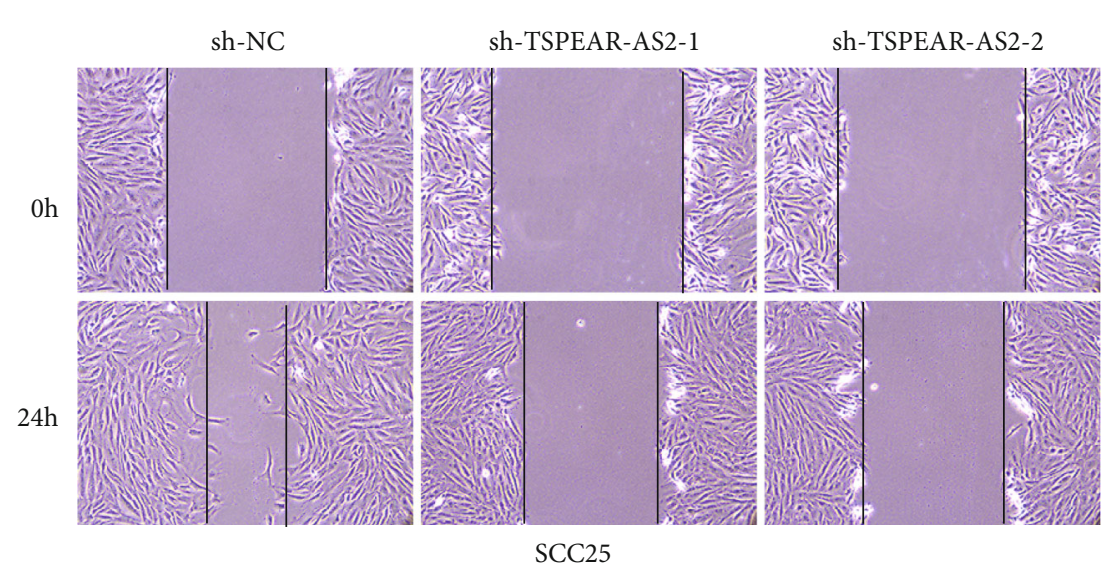

(b)

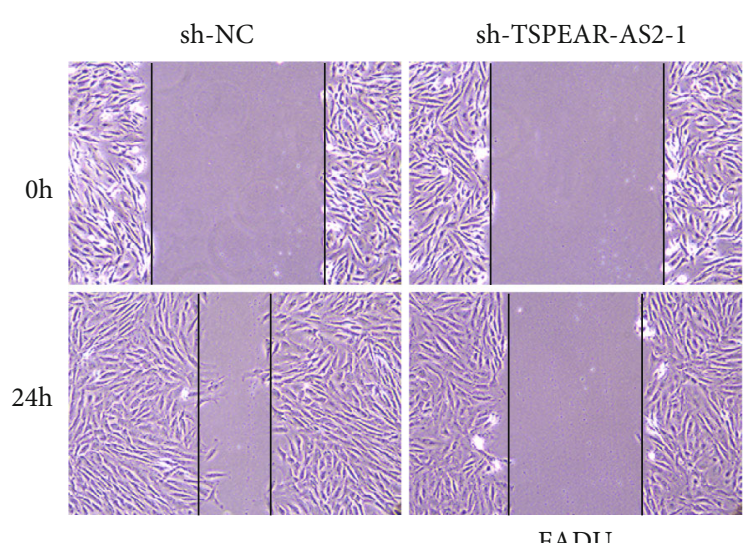

FADU

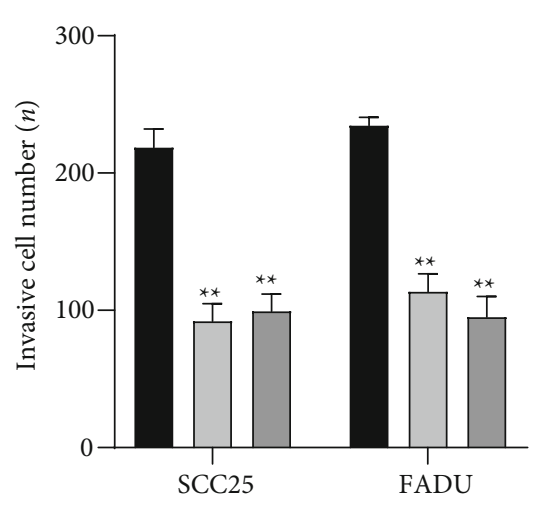

sh-NC

sh-TSPEAR-AS2-1

sh-TSPEAR-AS2-2
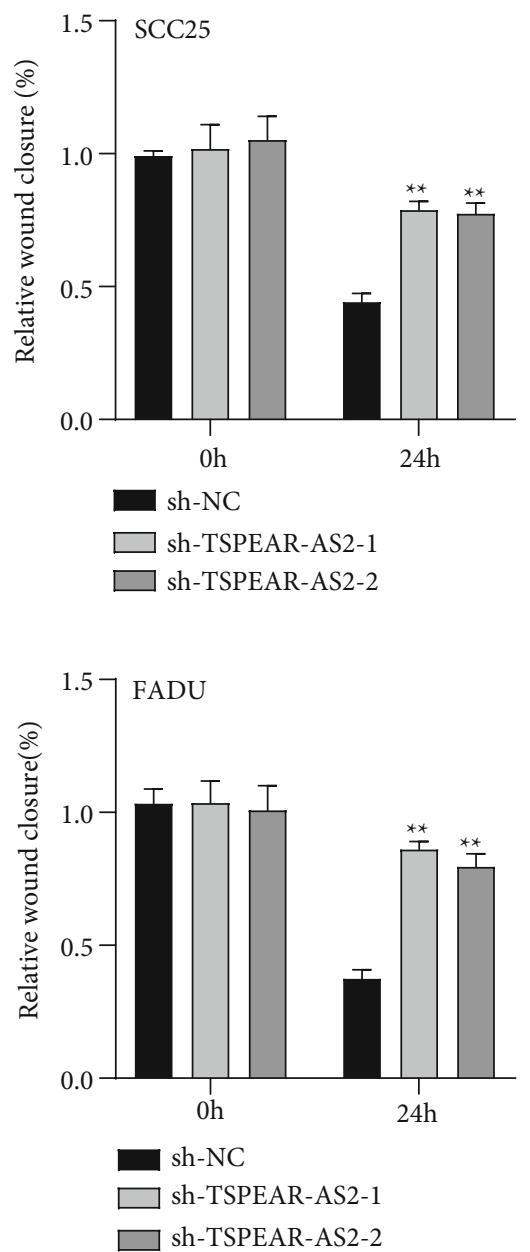

(c)

FIGURE 3: Knockdown of TSPEAR-AS2 suppressed the migrating and invading processes of OSCC cell. (a) Cell migration ability was detected in SCC25 and FADU cells in which TSPEAR-AS2 expression was decreased. (b, c) Transwell assay showed that knockdown of TSPEAR-AS2 could regulate SCC25 and FADU cells' invasive. ${ }^{* *} P<0.01$.

expression of PPM1A, while miR-487a-3p knockdown displayed an opposite effect. Based on the oncogenic roles of PPM1A in OSCC progression, we supposed that miR-487a$3 p$ may serve as a tumor suppressor via targeting PPM1A.
Finally, we performed rescue experiments, finding that knockdown of miR-487a-3p distinctly reversed the suppression of TSPEAR-AS2 knockdown on the expression of PPM1A protein. Thus, our findings suggested that TSPEAR-AS2 may 

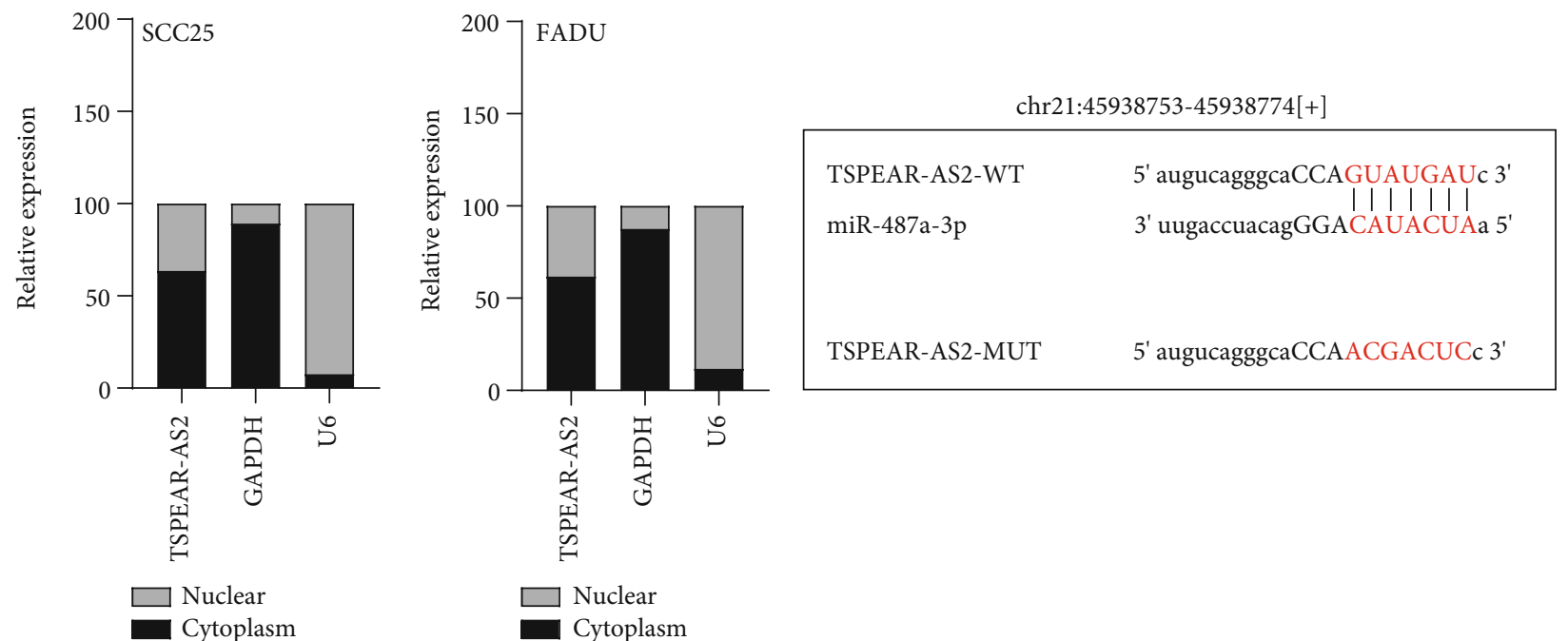

(a)

(b)
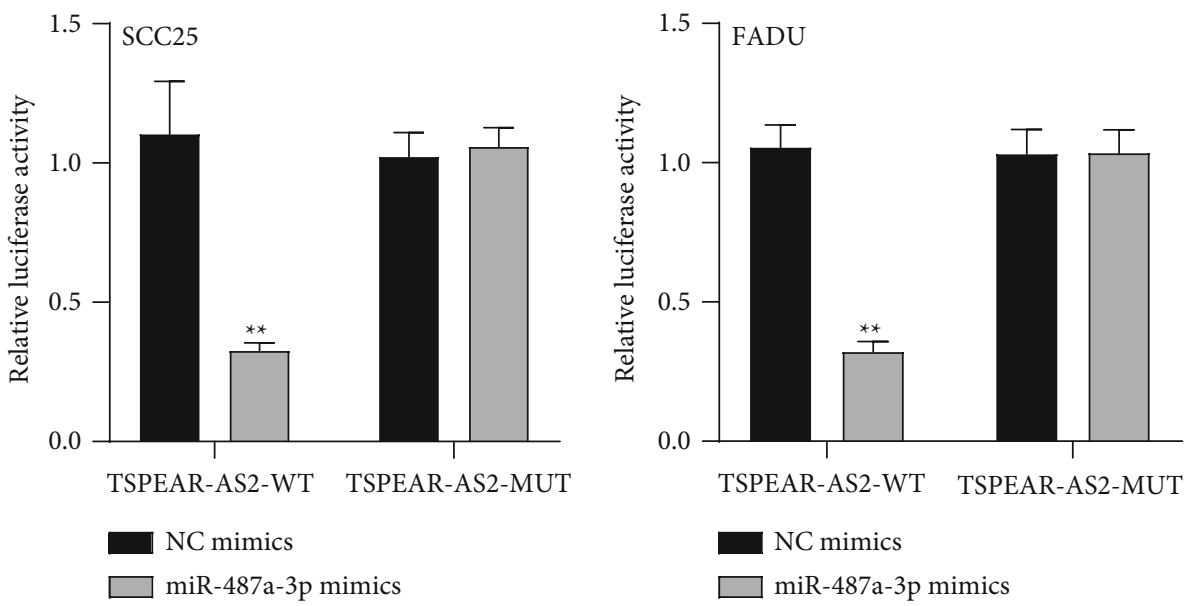

(c)

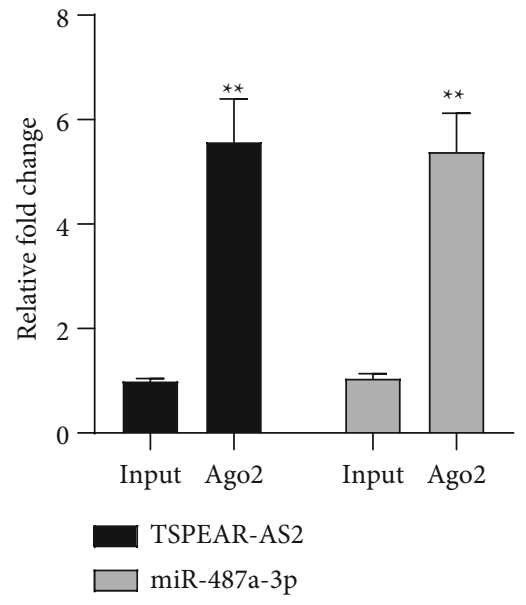

(d)

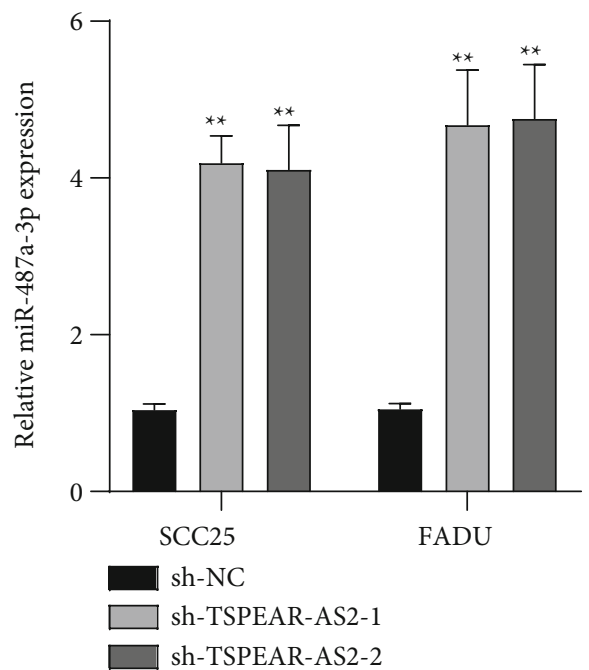

(e)

FIgURE 4: TSPEAR-AS2 acts as a molecular sponge of miR-487a-3p. (a) Relative TSPEAR-AS2 expression levels in nuclear and cytosolic fractions of SCC25 and FADU cells. (b) The binding sites of TSPEAR-AS2 with miR-487a-3p by Starbase 2.0. (c) According to luciferase reporter tests, miR-487a-3p reduced the luciferase activity of TSPEAR-AS2-WT, other than of TSPEAR-AS2-MUT. (d) RNA pull-down assays. (e) RT-PCR for miR-487a-3p expressing state in SCC25 and FADU cells after TSPEAR-AS2 knockdown. ${ }^{* *} P<0.01$. 


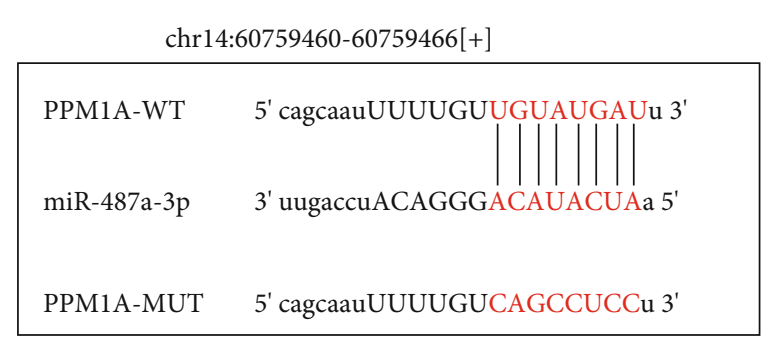

(a)

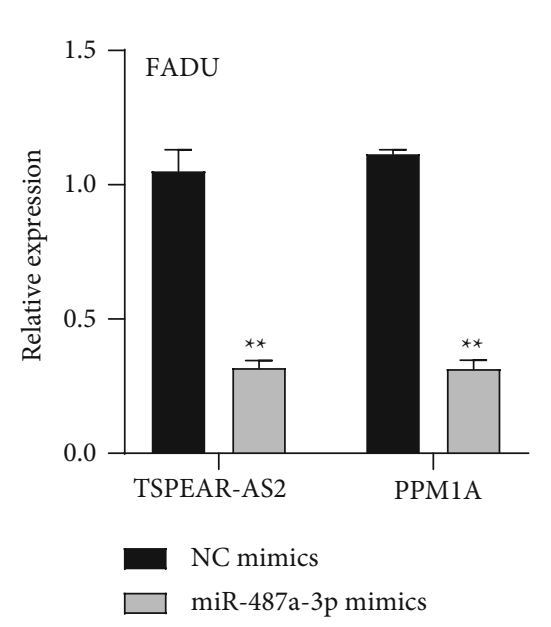

(c)

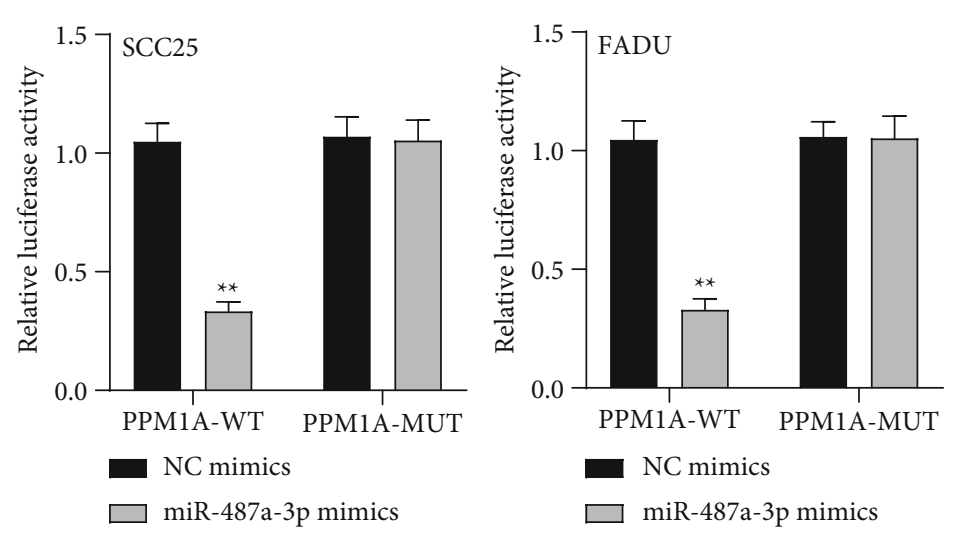

(b)

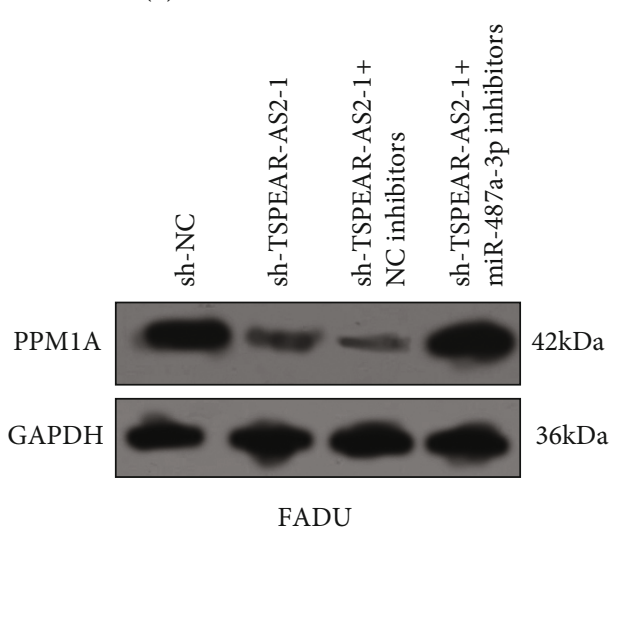

(d)

FIGURE 5: PPM1A was miR-487a-3p direct target gene. (a) Direct binding sites in miR-487a-3p and PPM1A were presented. (b) Luciferase reporter assay validated the molecular binding. (c) RT-PCR for the expressing states of miR-487a-3p and TSPEAR-AS2 in FADU cells after miR-487a-3p overexpression or knockdown. (d) Western blot assays determined the expression of PPM1A in FADU cells under the transfection by using sh-NC, sh-TSPEAR-AS2-1, sh-TSPEAR-AS2-1+ NC inhibitors, or sh-TSPEAR-AS2-1+ miR-487a-3p inhibitors. ${ }^{* *} P<0.01$.

display its suppression on the abilities of the proliferating, migrating, and invading processes pertaining to OSCC cell by increasing PPM1A expressing level by sponging miR-487a-3p.

\section{Conclusion}

To sum up, we identified TSPEAR-AS2 as a tumor-driver within OSCC, and the greater expressing state of TSPEARAS2 showed a relationship to tumor metastasis and poor prognosis. TSPEAR-AS2/miR-487a-3p/PPM1A axis may act as a new ceRNA regulatory network, thus, accelerating the malignant processes of OSCC. TSPEAR-AS2 may become a novel biomarker and therapeutically related target for this disease in the future.

\section{Data Availability}

The data used to support the findings of this study are available from the corresponding author upon request.

\section{Conflicts of Interest}

The authors declare no conflicts of interest.

\section{Acknowledgments}

This work was supported by grants from The high-level personnel Project of Yunnan Province (No. H2019016) and Kunming medical university union special project [No. 2017FE467-(156)].

\section{References}

[1] E. C. Smyth, J. Lagergren, R. C. Fitzgerald et al., "Oesophageal cancer," Nature Reviews Disease Primers, vol. 3, no. 1, article 17048, 2017.

[2] A. W. Y. Chai, K. P. Lim, and S. C. Cheong, "Translational genomics and recent advances in oral squamous cell carcinoma," Seminars in Cancer Biology, vol. 61, pp. 71-83, 2020.

[3] M. Irfan, R. Z. R. Delgado, and J. Frias-Lopez, "The oral microbiome and cancer," Frontiers in Immunology, vol. 11, article 591088, 2020. 
[4] M. Zhang, J. Liang, Y. Yang, H. Liang, H. Jia, and D. Li, "Current trends of targeted drug delivery for oral cancer therapy," Frontiers in Bioengineering and Biotechnology, vol. 8, article 618931, 2020.

[5] F. Ardito, G. Di Gioia, M. R. Pellegrino, and L. L. Muzio, "Genistein as a potential anticancer agent against head and neck squamous cell carcinoma," Current topics in Medicinal Chemistry, vol. 18, no. 3, pp. 174-181, 2018.

[6] Y. Lu, Z. Zheng, Y. Yuan et al., "The emerging role of exosomes in oral squamous cell carcinoma," Frontiers in Cell and Developmental Biology, vol. 9, article 628103, 2021.

[7] T. Sasahira and T. Kirita, "Hallmarks of cancer-related newly prognostic factors of oral squamous cell carcinoma," International Journal of Molecular Sciences, vol. 19, no. 8, article 2413, 2018.

[8] R. A. Boon, N. Jaé, L. Holdt, and S. Dimmeler, "Long noncoding RNAs: from clinical genetics to therapeutic targets?," Journal of the American College of Cardiology, vol. 67, no. 10, pp. 1214-1226, 2016.

[9] Y. H. Xing and L. L. Chen, "Processing and roles of snoRNA-ended long noncoding RNAs," Critical Reviews in Biochemistry and Molecular Biology, vol. 53, no. 6, pp. 596606, 2018.

[10] A. A. Farooqi, R. Attar, I. M. Yulaevna, and R. Berardi, "Interaction of long non-coding RNAs and circular RNAs with microRNAs for the regulation of immunological responses in human cancers," Seminars in Cell \& Developmental Biology, vol. S1084-9521, no. 21, article 00138-5, 2021.

[11] W. X. Peng, P. Koirala, and Y. Y. Mo, "LncRNA-mediated regulation of cell signaling in cancer," Oncogene, vol. 36, no. 41, pp. 5661-5667, 2017.

[12] M. Winkle, S. M. El-Daly, M. Fabbri, and G. A. Calin, "Noncoding RNA therapeutics - challenges and potential solutions," Nature Reviews Drug Discovery, pp. 1-23, 2021.

[13] Y. Chen, X. Zhou, C. Huang et al., "LncRNA PART1 promotes cell proliferation and progression in non-small-cell lung cancer cells via sponging miR-17-5p," Journal of Cellular Biochemistry, vol. 122, no. 3-4, pp. 315-325, 2021.

[14] B. Lin, H. He, Q. Zhang et al., "Long non-coding RNA00844 inhibits MAPK signaling to suppress the progression of hepatocellular carcinoma by targeting AZGP1," Annals of Translational Medicine, vol. 8, no. 21, article 1365, 2020.

[15] H. Che, Y. Che, Z. Zhang, and Q. Lu, "Long non-coding RNA LINC01929 accelerates progression of oral squamous cell carcinoma by targeting the miR-137-3p/FOXC1 axis," Frontiers in Oncology, vol. 11, article 657876, 2021.

[16] S. Chen, H. Xu, F. Hu, and T. Wang, "Identification of key players involved in $\mathrm{CoCl} 2$ hypoxia induced pulmonary artery hypertension in vitro," Frontiers in Genetics, vol. 11, p. 232, 2020.

[17] Z. H. Ma, Y. Shuai, X. Y. Gao et al., "BTEB2-activated lncRNA TSPEAR-AS2 drives GC progression through suppressing GJA1 expression and upregulating CLDN4 expression," Molecular Therapy Nucleic Acids, vol. 22, pp. 1129-1141, 2020.

[18] B. Xiu, Y. Chi, L. Liu et al., "LINC02273 drives breast cancer metastasis by epigenetically increasing AGR2 transcription," Molecular Cancer, vol. 18, no. 1, p. 187, 2019.

[19] Y. Tay, J. Rinn, and P. P. Pandolfi, “The multilayered complexity of ceRNA crosstalk and competition," Nature, vol. 505, no. 7483, pp. 344-352, 2014.
[20] J. J. Chan and Y. Tay, "Noncoding RNA:RNA regulatory networks in cancer," International Journal of Molecular Sciences, vol. 19, no. 5, article 1310, 2018.

[21] A. B. Santosh, T. Jones, and J. Harvey, "A review on oral cancer biomarkers: understanding the past and learning from the present," Journal of Cancer Research and Therapeutics, vol. 12, no. 2, pp. 486-492, 2016.

[22] R. Dumache, "Early diagnosis of oral squamous cell carcinoma by salivary microRNAs," Clinical Laboratory, vol. 63, no. 11, pp. 1771-1776, 2017.

[23] L. Liu, J. Chen, X. Cai, Z. Yao, and J. Huang, "Progress in targeted therapeutic drugs for oral squamous cell carcinoma," Surgical Oncology, vol. 31, pp. 90-97, 2019.

[24] C. Tomasovic-Loncaric, A. Fucic, A. Andabak et al., "Androgen receptor as a biomarker of oral squamous cell carcinoma progression risk," Anticancer Research, vol. 39, no. 8, pp. 4285-4289, 2019.

[25] F. Momen-Heravi and S. Bala, "Emerging role of non-coding RNA in oral cancer," Cellular Signalling, vol. 42, pp. 134$143,2018$.

[26] G. Z. Huang, Q. Q. Wu, Z. N. Zheng, T. R. Shao, and X. Z. Lv, "Identification of candidate biomarkers and analysis of prognostic values in oral squamous cell carcinoma," Frontiers in Oncology, vol. 9, article 1054, 2019.

[27] X. Niu, B. Yang, F. Liu, and Q. Fang, "LncRNA HOXA11-AS promotes OSCC progression by sponging miR-98-5p to upregulate YBX2 expression," Biomedicine \& Pharmacotherapy, vol. 121, article 109623, 2020.

[28] Y. Yang, D. Chen, H. Liu, and K. Yang, "Increased expression of lncRNA CASC9 promotes tumor progression by suppressing autophagy-mediated cell apoptosis via the AKT/mTOR pathway in oral squamous cell carcinoma," Cell Death \& Disease, vol. 10, no. 2, p. 41, 2019.

[29] C. Zhang, C. Bao, X. Zhang, X. Lin, D. Pan, and Y. Chen, "Knockdown of lncRNA LEF1-AS1 inhibited the progression of oral squamous cell carcinoma (OSCC) via hippo signaling pathway," Cancer Biology \& Therapy, vol. 20, no. 9, pp. 1213-1222, 2019.

[30] L. Statello, C. J. Guo, L. L. Chen, and M. Huarte, "Gene regulation by long non-coding RNAs and its biological functions," Nature Reviews Molecular Cell Biology, vol. 22, no. 2, pp. 96118, 2021.

[31] L. Chen, Y. H. Zhang, G. Lu, T. Huang, and Y. D. Cai, "Analysis of cancer-related lncRNAs using gene ontology and KEGG pathways," Artificial Intelligence in Medicine, vol. 76, pp. 27-36, 2017.

[32] S. W. Fu, Y. Zhang, S. Li, Z. Y. Shi, J. Zhao, and Q. L. He, "LncRNA TTN-AS1 promotes the progression of oral squamous cell carcinoma via miR-411-3p/NFAT5 axis," Cancer Cell International, vol. 20, no. 1, p. 415, 2020.

[33] H. Li and J. Jiang, "LncRNA MCM3AP-AS1 promotes proliferation, migration and invasion of oral squamous cell carcinoma cells via regulating miR-204-5p/FOXC1," Journal of Investigative Medicine, vol. 68, no. 7, pp. 1282-1288, 2020.

[34] Y. Cai, X. Yu, S. Hu, and J. Yu, "A brief review on the mechanisms of miRNA regulation," Genomics, Proteomics \& Bioinformatics, vol. 7, no. 4, pp. 147-154, 2009.

[35] A. Tiwari, B. Mukherjee, and M. Dixit, "MicroRNA key to angiogenesis regulation: miRNA biology and therapy," Current Cancer Drug Targets, vol. 18, no. 3, pp. 266-277, 2018. 
[36] Z. Wang, X. Zhu, P. Dong, and J. Cai, "Long noncoding RNA LINC00958 promotes the oral squamous cell carcinoma by sponging miR-185-5p/YWHAZ," Life Sciences, vol. 242, article 116782, 2020.

[37] X. Jiang, J. Liu, S. Li et al., "CCL18-induced LINC00319 promotes proliferation and metastasis in oral squamous cell carcinoma via the miR-199a-5p/FZD4 axis," Cell Death \& Disease, vol. 11, no. 9, p. 777, 2020.

[38] Y. Sun, Z. Cao, J. Shan et al., "Hsa_circ_0020095 promotes oncogenesis and cisplatin resistance in colon cancer by sponging miR-487a-3p and modulating SOX9," Frontiers in Cell and Developmental Biology, vol. 8, article 604869, 2021.

[39] X. Yang, M. Wang, B. Lin et al., "miR-487a promotes progression of gastric cancer by targeting TIA1," Biochimie, vol. 154, pp. 119-126, 2018.

[40] L. Wang, S. Ge, and F. Zhou, "MicroRNA-487a-3p inhibits the growth and invasiveness of oral squamous cell carcinoma by targeting PPM1A," Bioengineered, vol. 12, no. 1, pp. 937-947, 2021.

[41] S. R. Smith, K. Schaaf, N. Rajabalee et al., "The phosphatase PPM1A controls monocyte-to-macrophage differentiation," Scientific Reports, vol. 8, no. 1, p. 902, 2018.

[42] K. W. Khim, S. S. Choi, H. J. Jang et al., "PPM1A controls diabetic gene programming through directly dephosphorylating PPAR $\gamma$ at Ser273," Cells, vol. 9, no. 2, p. 343, 2020.

[43] Y. Hong, L. Gong, B. Yu, and Y. Dong, "PPM1A suppresses the proliferation and invasiveness of RCC cells via Smad2/3 signaling inhibition," Journal of Receptor and Signal Transduction Research, vol. 41, no. 3, pp. 245-254, 2021. 